\title{
Streaming-to-accumulation transition in a two-dimensional electron system in a polar semiconductor
}

\author{
W. Xu, F. M. Peeters, and J. T. Devreese* \\ Department of Physics, University of Antwerp (U.I.A.), Universiteitsplein 1, B-2610 Antwerpen, Belgium
}

(Received 3 February 1992)

\begin{abstract}
Hot-electron transport is studied for a two-dimensional electron gas coupled to longitudinal-optical phonons in crossed electric and magnetic fields. At low electric and high magnetic fields the electrons are accumulated, while at high electric fields they are in a streaming state. We develop a streaming-toaccumulation transition model and compare the results with that from a Monte Carlo simulation.
\end{abstract}

\section{INTRODUCTION}

The investigation of nonlinear electron (or hotelectron) transport has played an important role in modern semiconductor physics. ${ }^{1}$ The interest in this topic was stimulated both by basic semiconductor physics and by applications in electronic devices. ${ }^{2}$ Hot-electron phenomena in bulk materials ${ }^{3}$ are primarily determined by (1) the band structure and (2) electron-phonon scattering processes. With the development and applications of high electron mobility transistor (HEMT) structures, ${ }^{4}$ hot-electron transport in a two-dimensional system has been a fruitful subject both for theory ${ }^{5}$ and experiment. ${ }^{6}$ In a quasi-two-dimensional electron system one would expect different nonlinear transport effects from the corresponding three-dimensional (3D) case. The basic differences of electron transport in 2D and 3D arise from (1) the electron wave functions and density of states, which are different in quantum-confined systems; ${ }^{7}$ (2) the interactions of electrons with scatters in a 2D system, which have different ${ }^{8}$ character than those in $3 \mathrm{D}$; and (3) the fact that for a 2D system the confinement of the electron's motion in the $z$ direction implies that the momentum in this direction will no longer be conserved ${ }^{9}$ during a scattering event, which leads to an enhancement of the effective electron-phonon scattering interaction ${ }^{10}$ and consequently to a lowering of the mobility.

For a polar semiconductor like GaAs, the limiting factor determining the electronic mobility at high temperature, i.e., $T>40 \mathrm{~K}, \quad$ is the process of electron-longitudinal-optical (LO) -phonon scattering. ${ }^{11}$ At high electric fields LO-phonon scattering becomes important $^{12}$ at even lower temperatures because the electron is able to gain sufficient energy to emit a LO phonon. Due to the inelastic nature of the electron-LO-phonon scattering (1) it leads to highly nonlinear electron transport, and (2) it is very difficult to find reliable approximate solutions to the transport equation which in most situations is the Boltzmann equation. ${ }^{13}$ Therefore, we consider the extreme situation in which this electronphonon scattering mechanism is the only one which is active. ${ }^{14}$ The Monte Carlo method, ${ }^{15}$ which is a pure numerical technique and consequently very time consuming, turns out to be very suitable for our purpose. Such a study was performed by us for the 3D (Ref. 16) and the 2D (Ref. 17) case. Here we generalize it to 2D transport in the presence of a nonquantizing perpendicular magnetic field.

By applying a weak magnetic field (i.e., it is not strong enough to lead to Landau quantization) perpendicular to the electric field and to the $x y$ plane, we introduce a very specific motion of electrons and it is possible to obtain more information about the electron transport. For example, Hall measurements ${ }^{18}$ on polar semiconductors will reflect the type of electron motion ${ }^{19}$ and consequently the electron momentum distribution. ${ }^{20}$ The interpretation of the experimental results on very low electron density samples requires detailed knowledge of the Hall factor $\left(r_{H}\right)$, which is defined as the ratio of the Hall mobility $\mu_{H}$ to the drift mobility $\mu_{D}$.

To solve the Boltzmann equation for electrons coupled to LO phonons in a polar semiconductor, Devreese and Evrard proposed ${ }^{21}$ a "two-circle" model approximation for the case of electrons moving in 3D at zero temperature. Under the approximation of this "two-circle" model, the Boltzmann equation was solved quasianalytically (1) in Ref. 22 for a two-dimensional electron gas at zero temperature and (2) in Ref. 23 for the 3D case in the presence of a weak magnetic field. Komiyama, Masumi, and Kajita studied ${ }^{19}$ the magnetotransport in a 3D polar semiconductor such as $\mathrm{AgCl}$ and $\mathrm{AgBr}$. Their experiments have provided evidence for streaming motion and for electron accumulation induced by electron-LOphonon interaction in the case of crossed electric and magnetic fields. At sufficiently high electric fields or relatively low temperature, the conducting electrons in a polar semiconductor perform a streaming motion: ${ }^{24}$ the average streaming velocity of electrons depends very little on the electric field and is equal to $v_{s} \sim v_{\mathrm{LO}} / 2$ with $v_{\mathrm{LO}}=\left(2 \hbar \omega_{\mathrm{LO}} / m^{*}\right)^{1 / 2}$. When a sufficiently strong magnetic field perpendicular to the electric field is applied, the electrons are forced into a closed orbit. The electrons are accumulated ${ }^{25}$ in a region in momentum space with $v<v_{\text {LO }}$ and are unable to gain sufficient energy to emit a LO phonon. The streaming-to-accumulation transition was studied $^{26}$ by Warmenbol, Peeters, and Devreese for a bulk polar semiconducting material. Since the electron-LO-phonon interaction in a $2 \mathrm{D}$ system $^{17}$ is different from the corresponding 3D case, and electron momentum in the $z$ direction is no longer conserved for the $2 \mathrm{D}$ case, one expects that the streaming and accumulation motions of electrons in a two-dimensional system 
should exhibit differences from that in a bulk material.

In this present paper we study the streaming-toaccumulation transition for a two-dimensional electron system in crossed electric and magnetic fields. In order to limit the number of parameters, we consider the extreme situation of an ideal two-dimensional electron system (I2DEG), ${ }^{27}$ i.e., the width of the $2 \mathrm{D}$ layer is taken to be zero. In this paper we will limit ourselves to the case where the electrons are only scattered by LO phonons. As an exact solution of the Boltzmann equation for the model, the Monte Carlo simulation is described in Sec. II. In Sec. III the model of streaming-to-accumulation transition is constructed for two-dimensional electron transport and the results are compared with the Monte Carlo calculations. Our conclusions are summarized in Sec. IV.

\section{MONTE CARLO SIMULATION}

In the present paper we employ the single electron Monte Carlo simulation technique ${ }^{15}$ to study the electron motion in a two-dimensional system in crossed electric and magnetic fields. For simplicity we assume a parabolic conduction band and neglect the finite extent of the 2D electron layer. The equation of motion of an electron during a free flight is (in cgs units)

$$
m * \frac{d \mathbf{v}}{d t}=-e(\mathbf{E}+\mathbf{v} \times \mathbf{B}),
$$

where $\mathbf{E}$ is the electric field, $\mathbf{B}$ the magnetic field, and $\mathbf{v}$ the electron velocity. For the case of $E=(E, 0,0)$ and $\mathbf{B}=(0,0, B)$, and for a parabolic band structure, the solution of Eq. (1) is

$$
\begin{aligned}
& v_{x}(t)=v_{x}(0) \cos \left(\omega_{c} t\right)- {\left[v_{y}(0)+E / B\right] \sin \left(\omega_{c} t\right), } \\
& v_{y}(t)=v_{x}(0) \sin \left(\omega_{c} t\right)+ {\left[v_{y}(0)+E / B\right] } \\
& \times \cos \left(\omega_{c} t\right)-E / B,
\end{aligned}
$$

where $\left[v_{x}(0), v_{y}(0)\right]$ is the velocity of the electron at time $t=0$, and $\omega_{c}=e B / m^{*}$ is the usual cyclotron frequency with effective mass $m^{*}$. Notice that during the free flight energy is conserved

$\left[v_{x}(0)\right]^{2}+\left[v_{y}(0)+\frac{E}{B}\right]^{2}=\left[v_{x}(t)\right]^{2}+\left[v_{y}(t)+\frac{E}{B}\right]^{2}$

In a single electron Monte Carlo simulation, the duration of a free flight for the sample electron is determined by the scattering rates. For electron-LO-phonon coupling in a $2 \mathrm{D}$ system the transition rate per unit time $P\left(\mathbf{p}^{\prime}, \mathbf{p}\right)$ for an electron to scatter from state $\mathbf{p}^{\prime}$ to $\mathbf{p}$ is

$$
\begin{aligned}
P_{ \pm}\left(\mathbf{p}^{\prime}, \mathbf{p}\right)= & \alpha L_{0}\left(\hbar \omega_{\mathrm{LO}}\right)^{2}\left[\begin{array}{c}
N_{0} \\
N_{0}+1
\end{array}\right] \\
& \times \frac{\delta\left(p^{\prime 2} / 2 m^{*}-p^{2} / 2 m^{*} \mp \hbar \omega_{\mathrm{LO}}\right)}{\left|\mathbf{p}^{\prime}-\mathbf{p}\right|},
\end{aligned}
$$

where $\alpha$ is the electron-LO-phonon coupling constant ( $\alpha=0.068$ for GaAS), $\hbar \omega_{\mathrm{LO}}$ is the energy of a LO phonon $\left(\hbar \omega_{\mathrm{LO}}=36.6 \mathrm{meV}\right.$ for GaAs), $L_{0}=\left(\hbar / 2 m^{*} \omega_{\mathrm{LO}}\right)^{1 / 2}$ is the unit of length $\left(L_{0}=39.5 \AA\right.$ for GaAs), $N_{0}=1 /\left(e^{\hbar \omega_{\mathrm{LO}} / k_{B} T}-1\right)$ is the LO-phonon occupation number, and the plus (minus) sign refers to absorption (emission) of a LO phonon. The scattering rate for an electron with a kinetic energy $E=x \hbar \omega_{\text {LO }}$ then becomes

$$
\lambda_{ \pm}(x)=2 \alpha \omega_{\mathrm{LO}} \times\left\{\begin{array}{l}
N_{0} \Theta(x) K(\sqrt{x /(x+1)}) / \sqrt{x+1} \text { for absorption } \\
\left(N_{0}+1\right) \Theta(x-1) K(\sqrt{(x-1) / x}) / \sqrt{x} \text { for emission }
\end{array}\right.
$$

where $\Theta(x)=0(x<0), 1(x \geq 0)$ is the unit-step function, and $K(x)$ is the complete elliptic integral of the first kind. The probability to scatter the electron over an angle $0 \leq \theta \leq 2 \pi$ when its initial momentum is $\mathbf{p}$ and its final momentum is $\mathbf{p}^{\prime}$ is

$$
P(\theta) \sim \frac{1}{\sqrt{p^{\prime 2}+p^{2}-2 p^{\prime} p \cos \theta}} .
$$

After normalizing Eq. (6a) and defining $S=2 \sqrt{E E^{\prime}} /\left(\sqrt{E}-\sqrt{E^{\prime}}\right)^{2}$ with $E=p^{2} / 2 m^{*}$ and $E^{\prime}=p^{\prime 2} / 2 m^{*}$, we obtain

$$
P(\theta)=\frac{1}{4}\left[\frac{1+2 S}{1+S(1-\cos \theta)}\right]^{1 / 2} / K\left[\left(\frac{2 S}{1+2 S}\right]^{1 / 2}\right] .
$$

The results of our Monte Carlo simulation for the electron momentum-distribution function are shown in Figs. 1(a) -1 (d) at a lattice temperature of $T=55 \mathrm{~K}$ and a fixed magnetic field of $B=50 \mathrm{G}$ for different electric-field values. Note that a dimensionless quantity $\zeta=v_{\mathrm{LO}} \boldsymbol{B} / \boldsymbol{E}$, with $v_{\mathrm{LO}}=\left(2 \hbar \omega_{\mathrm{LO}} / m^{*}\right)^{1 / 2}$ the unit of velocity $\left(v_{\mathrm{LO}}=4.40 \times 10^{7} \mathrm{~cm} / \mathrm{s}\right.$ for GaAs), specifies the position of the center of the cyclotron orbit $(0,-1 / \zeta)$ in velocity space relative to the polaron circle $|\mathbf{v}|=v_{\text {LO }}$. Inside this circle, only LO-phonon absorption occurs and LOphonon emission is impossible. Outside this circle LOphonon emission is the dominant scattering process. The results show that (1) for low electric fields, i.e., $\zeta>2$, the magnetic field is sufficiently strong to force the electron in a circular orbit within the polaron circle. The electrons are accumulated on closed orbits which is interrupted only through absorption of a LO phonon. The electron distribution at very low temperature is centered around $\left(0,-v_{\mathrm{LO}} / \xi\right)$ but at higher temperatures, due to LO-phonon scattering, it also acquires a component along the electric field; (2) for intermediate electric fields, $1<\zeta<2$, part of the electrons are streaming and another part of the electrons are accumulated on closed orbits as is shown in Figs. 1(b) and 1(c). This is the streaming-toaccumulation transition regime; and (3) for high electric fields, i.e., $\zeta<1$, the influence of the electric field is dom- 

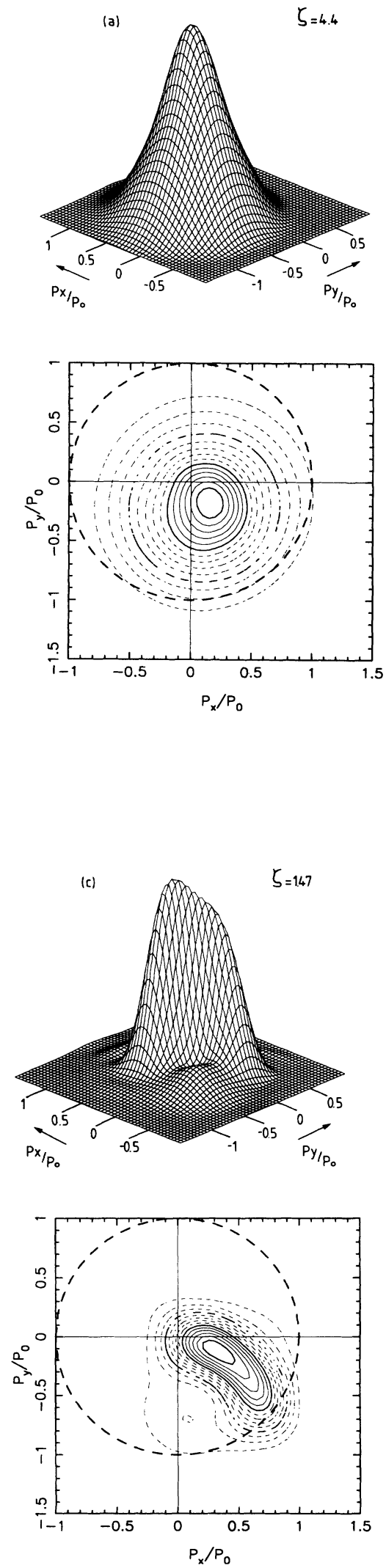
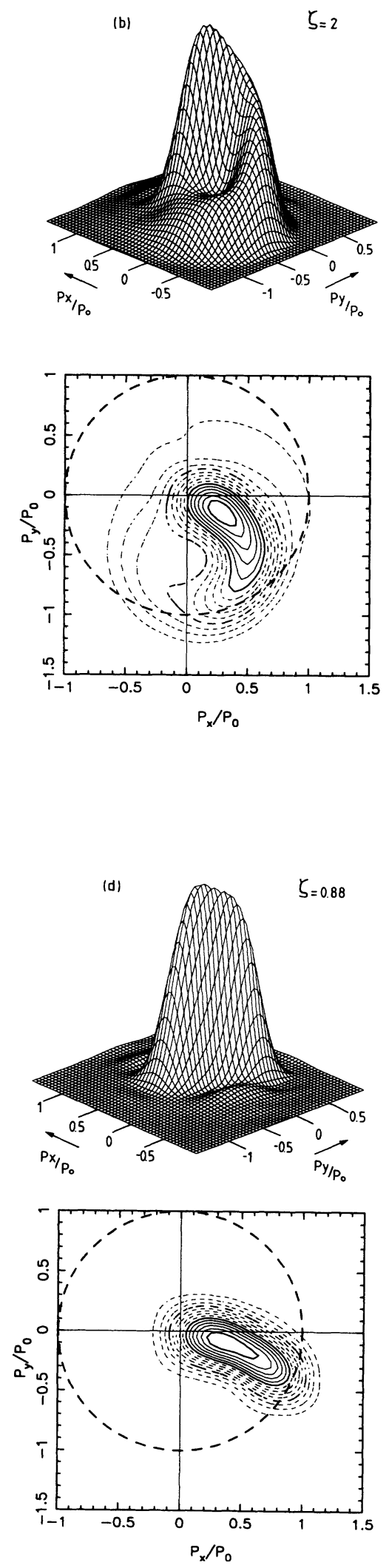

FIG. 1. Electron momentum-distribution function $f\left(p_{x}, p_{y}\right)$ and the corresponding contour plot at a fixed magnetic field of $B=50$ $\mathrm{G}$ and at temperature of $T=55 \mathrm{~K}$ for different electric fields: (a) small electric field $E=5 \mathrm{~V} / \mathrm{cm}$; (b) intermediate field $E=11 \mathrm{~V} / \mathrm{cm}$ and (c) $E=15 \mathrm{~V} / \mathrm{cm}$; and (d) high field $E=25 \mathrm{~V} / \mathrm{cm} . P_{0}=\left(2 \hbar \omega_{\mathrm{LO}} m^{*}\right)^{1 / 2}$ is the unit of momentum $\left(P_{0}=2.67 \times 10^{-21} \mathrm{~g} \mathrm{~cm} / \mathrm{s}\right.$ for $\mathrm{GaAs}) . \zeta=v_{\mathrm{LO}} B / E$ with $v_{\mathrm{LO}}$ the unit of velocity $\left(v_{\mathrm{LO}}=4.4 \times 10^{7} \mathrm{~cm} / \mathrm{s}\right.$ for GaAs). The dashed circle (in a thicker line width) is the polar circle $P=P_{0}$. 
inant and the electrons are in the streaming state, as shown in Fig. 1(d). The effect of the magnetic field is to deflect the electron motion slightly from a straight motion into a curved orbit. In this regime the LOphonon emission is the dominant scattering mechanism and the electrons are able to penetrate slightly into the active region.

Experimentally one usually measures average quantities like velocities, electron temperatures, Hall factor, etc. For a free flight of duration $\tau$ we obtain the average electron velocities

$$
\begin{aligned}
& \left\langle v_{x}\right\rangle=\frac{1}{\tau} \int_{0}^{\tau} d t v_{x}(t)=\frac{v_{y}(\tau)-v_{y}(0)}{\omega_{c} \tau} \\
& \left\langle v_{y}\right\rangle=\frac{1}{\tau} \int_{0}^{\tau} d t v_{y}(t)=-\frac{E}{B}-\frac{v_{x}(\tau)-v_{x}(0)}{\omega_{c} \tau}
\end{aligned}
$$

and the average kinetic energies

$$
\begin{aligned}
\left\langle v_{x}^{2}\right\rangle= & \frac{1}{\tau} \int_{0}^{\tau} d t v_{x}^{2}(t) \\
= & \frac{1}{2}\left[v_{x}^{2}(0)+\left[v_{y}(0)+\frac{E}{B}\right]^{2}\right] \\
& +\frac{v_{x}(\tau)\left[v_{y}(\tau)+E / B\right]-v_{x}(0)\left[v_{y}(0)+E / B\right]}{2 \omega_{c} \tau},
\end{aligned}
$$

and

$$
\begin{aligned}
\left\langle v_{y}^{2}\right\rangle=\frac{1}{\tau} \int_{0}^{\tau} d t v_{y}^{2}(t)= & \frac{1}{2}\left[v_{x}^{2}(0)+\left[v_{y}(0)+\frac{E}{B}\right]^{2}\right]+\left[\frac{E}{B}\right]^{2} \\
& +\frac{1}{2 \omega_{c} \tau}\left[v_{x}(0)\left(v_{x}(0)+\frac{E}{B}\right]-v_{x}(\tau)\left[v_{y}(\tau)+\frac{E}{B}\right]+\frac{4 E}{B}\left[v_{x}(\tau)-v_{x}(0)\right]\right]
\end{aligned}
$$

Then (1) the electron temperatures are defined by $T_{x}=m^{*}\left\langle v_{x}^{2}\right\rangle / k_{B}$ for electron motion in the $x$ direction and $T_{y}=m^{*}\left\langle v_{y}^{2}\right\rangle / k_{B}$ for electron motion in the $y$ direction; (2) the Hall factor (for a long sample and in the Hall configuration) is defined by ${ }^{28}$ by

$$
r_{H}=\frac{E}{B} \frac{\left|\left\langle v_{y}\right\rangle\right|}{\left\langle v_{x}\right\rangle^{2}+\left\langle v_{y}\right\rangle^{2}}
$$

and (3) the tangent of the Hall angle $\tan \left(\theta_{H}\right)=\left\langle v_{y}\right\rangle /\left\langle v_{x}\right\rangle$. As obtained from our Monte Carlo simulation, the electron average velocities, the electron temperatures, and the Hall factor and the tangent of the Hall angle are depicted in Figs. 2, 3, and 4 as a function of the strength of the electric field $1 / \zeta=E / B v_{\text {LO }}$ for (a) a fixed magnetic field of $B=50 \mathrm{G}$ and for different lattice temperatures, and (b) a fixed temperature of $T=60 \mathrm{~K}$, respectively, for different magnetic fields. The three regimes: (1) the accumulation regime $(\zeta>2)$; (2) the streaming-to-accumulation transition regime $(1<\zeta<2)$; and (3) the streaming regime $(\xi>2)$ have a clear distinct signature on the average electron velocities (Fig. 2), the electron temperatures (Fig. 3), the Hall factor and the tangent of the Hall angle (Fig. 4).

In the accumulation regime $(\zeta>2)$ : (1) $\left\langle v_{y}\right\rangle$ $\sim E / B \sim 1 / \zeta$ is linear in the electric field while $\left\langle v_{x}\right\rangle \sim 0$ [see Fig. 2(a)]. Increasing the lattice temperature will decrease $\left\langle v_{y}\right\rangle$ because of increased LO-phonon scattering, while $\left\langle v_{x}\right\rangle$ first increases because temperature randomizes the electron motion and thus tends to make the electron distribution more isotropic. With a further increase of temperature $\left\langle v_{x}\right\rangle$ also decreases because of enhanced scattering which lowers the average velocity. Increasing the magnetic field will increase $\left\langle v_{y}\right\rangle$ while $\left\langle v_{x}\right\rangle$ de- creases [see Fig. 2(b)], which is due to the fact that the magnetic field bends the path of electron motion toward the $y$ direction; (2) the electron temperature $T_{y}$ increases with electric field (see Fig. 3 ) because $\left\langle v_{y}\right\rangle$ increases and as a consequence the electron orbit radius increases, which leads to an increased kinetic energy. Because of the curvature of the path of the electrons motion by the Lorentz force, the rate at which electrons gain energy from the electric field (in the $x$ direction) is decreased, which leads to the cooling of the electron motion in the $x$ direction. Our Monte Carlo results in Fig. 3 show that the cooling of the electrons in the $x$ direction is more pronounced at low temperature and at high magnetic fields. Increasing the lattice temperature and (or) decreasing the magnetic field wash out these effects. This thermomagnetic hot-electron effect, induced by electron-LOphonon coupling, was expected by Cowell ${ }^{29}$ and described by Pozhela ${ }^{30}$ at strong magnetic field for a bulk semiconducting material; and (3) in the accumulation regime the Hall factor increases and the tangent of the Hall angle slightly decreases with increasing electric field (see Fig. 4), which results from both an increase of $\left\langle v_{x}\right\rangle$ and $\left\langle v_{y}\right\rangle$.

In the strong electric-field regime $(\zeta<1)$ the electrons are streaming: (1) $\left\langle v_{x}\right\rangle \sim v_{\text {LO }} / 2$, while $\left\langle v_{y}\right\rangle$ decreases with increasing electric field because the electron orbit is less deflected by the Lorentz force; (2) $T_{x}$ depends little on the fields and $T_{y} \rightarrow 0$. Increasing the lattice temperature tends to make $T_{x}$ and $T_{y}$ equal to $T$, which implies that $T_{x}$ decreases and $T_{y}$ increases as function of $T$; and (3) the Hall factor $r_{H}$ and the tangent of the Hall angle $\tan \left(\theta_{H}\right)$ stay almost constant with increasing electric field. 
The intermediate retime $(1<\zeta<2)$ is a transition regime between the above two regimes and here we see the strongest changes in the average velocities, the electron temperatures, the Hall factor, and the tangent of the Hall angle. At fixed temperature, increasing the magnetic field will sharpen the accumulation-to-streaming transition as is illustrated in Figs. 2(b), 3(b), and 4(b).

In Figs. 2(a), 3(a), and 4(a) we compare the Monte Car-
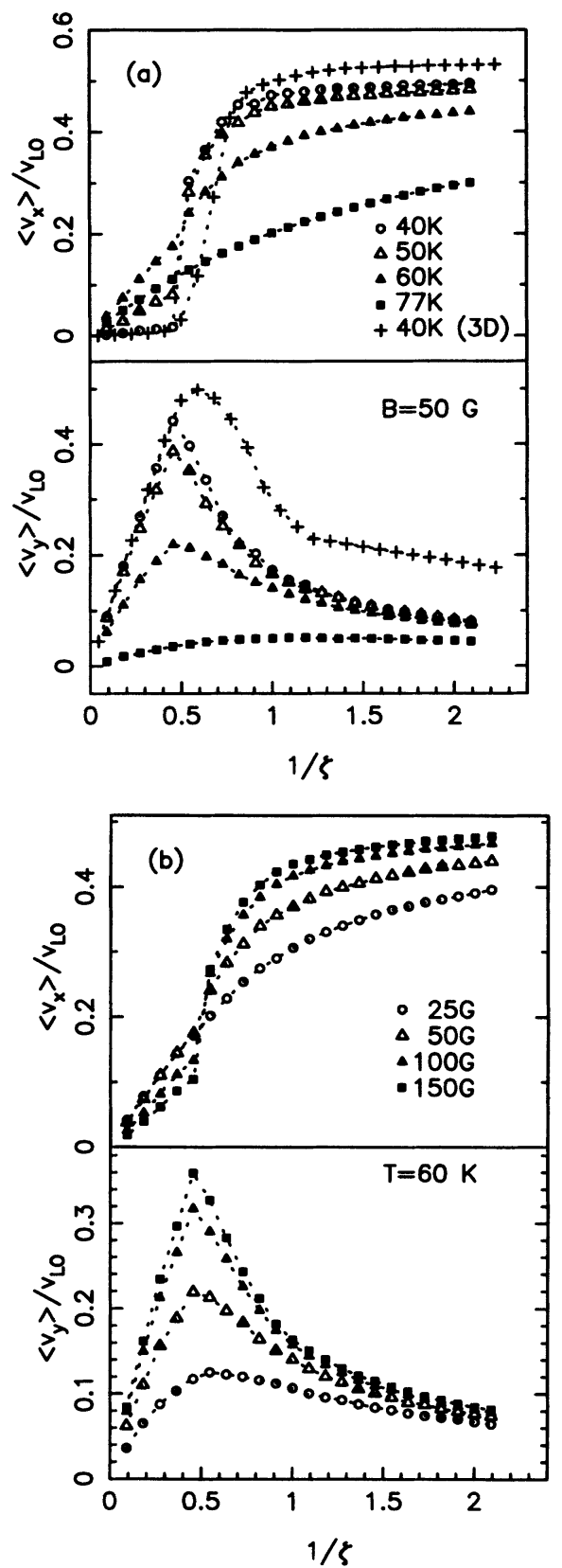

FIG. 2. The $x$ and $y$ components of the drift velocity as obtained from the present Monte Carlo simulation as a function of the electric field: (a) for a fixed magnetic field of $B=50 \mathrm{G}$ and different temperatures, and (b) for a fixed temperature of $T=60$ $\mathbf{K}$ and different magnetic fields. In (a) the results are compared with the 3D case (crosses). The symbols are connected by the dotted curves to guide the eye. $\zeta=v_{\mathrm{LO}} B / E$ with the unit of velocity $v_{\mathrm{LO}}=\left(2 \hbar \omega_{\mathrm{LO}} / \mathrm{m}^{*}\right)^{1 / 2}\left(v_{\mathrm{LO}}=4.4 \times 10^{7} \mathrm{~cm} / \mathrm{s}\right.$ for GaAs$)$. lo results for electron motion in two dimensions to the corresponding 3D case calculated in Ref. 26. A twodimensional electron gas has a more pronounced streaming-to-accumulation transition than the corresponding 3D case [compare the crosses in Figs. 2(a), 3(a), and 4(a) with the open circles] because (1) the LO-phonon emission scattering rate has a steeper increase around the electron energy $E \geq \hbar \omega_{\mathrm{LO}}$ in $2 \mathrm{D}$, and (2) the absence of the $z$ direction in the 2D case which in 3D tends to smoothen the transition. The LO-phonon emission scattering rate in $2 \mathrm{D}$ is always larger than ${ }^{31}$ for the $3 \mathrm{D}$ situation, the electron average velocity in the streaming regime is larger for $3 \mathrm{D}$ electrons.
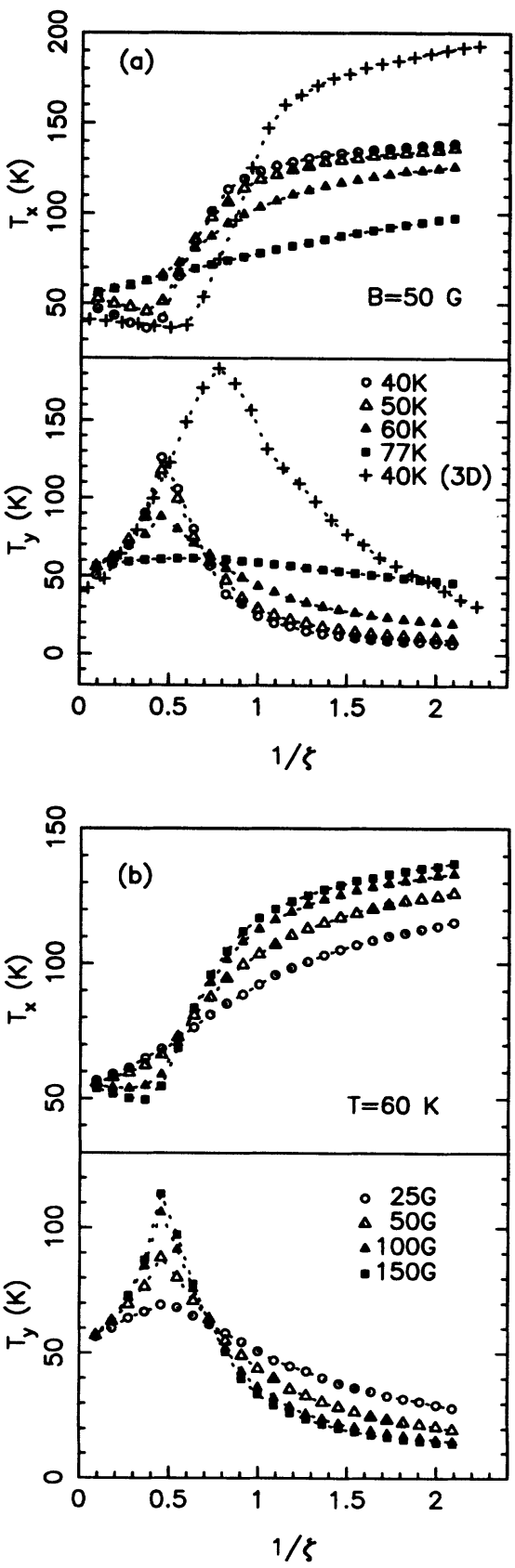

FIG. 3. The electron temperatures in the $x$ and $y$ direction as a function of the electric field as obtained from the present Monte Carlo simulation (same as in Fig. 2). 


\section{THE STREAMING-TO-ACCUMULATION MODEL}

In this section we first give some simple analyses for electron transport in crossed electric and magnetic fields. Then the streaming-to-accumulation transition model of Ref. 26 is developed for a two-dimensional electron gas. The results obtained with the present model will be compared with the results from our Monte Carlo simulation.

\section{A. Simple analyses for $B \rightarrow 0$ and $E \rightarrow 0$}

Since the Hall factor $r_{H}$, defined by the ratio of the Hall mobility $\mu_{H}$ to the drift mobility $\mu_{D}$, is a measure of
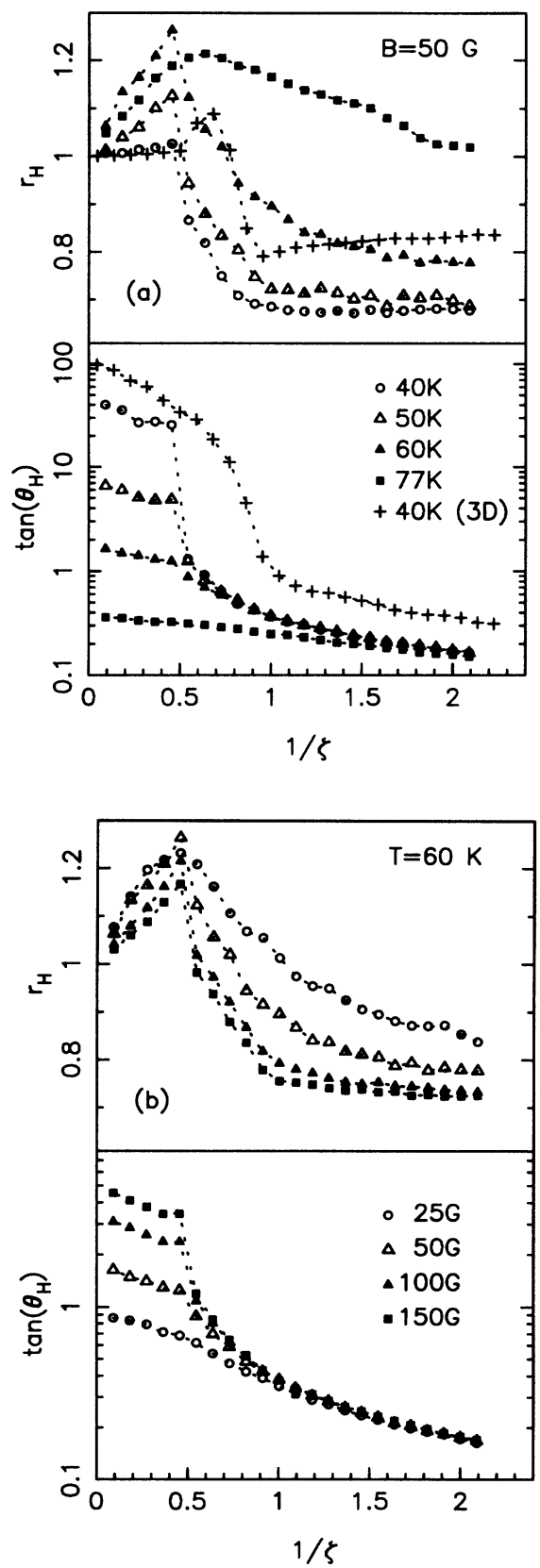

FIG. 4. The Hall factor $r_{H}$ and the tangent of the Hall angle $\tan \left(\theta_{H}\right)$ as a function of the electric field as obtained from the present Monte Carlo simulation (same as in Fig. 2). how much the drift and Hall mobility are different and therefore is of most importance for transport of electrons in crossed electric and magnetic fields, we first study the Hall factor for small electric and magnetic fields. In the limit of vanishing electric and magnetic fields the Hall factor takes the form ${ }^{28}$

$$
\lim _{B \rightarrow 0 E \rightarrow 0} \lim _{B} r_{H}=\frac{\left\langle\tau_{m}^{2}\right\rangle}{\left\langle\tau_{m}\right\rangle^{2}},
$$

where the averages for a $2 \mathrm{D}$ electron system are defined through

$$
\langle A\rangle=\int_{0}^{\infty} d y y A(y) e^{-y},
$$

with $y=E / k_{B} T$, and $1 / \tau_{m}=\lambda_{-}(E)+\lambda_{+}(E)$ is the inverse of the relaxation time for electron-LO-phonon scattering where $\lambda_{ \pm}(x)$ is given by Eq. (5). The numerical result for $r_{H}$ at $E=B \rightarrow 0$ is shown in Fig. 5 as a function of temperature for a very low electron density system. The unit of temperature is $\Theta_{D}=\hbar \omega_{\mathrm{LO}} / k_{B}$ $\left(\Theta_{D}=425 \mathrm{~K}\right.$ for $\left.\mathrm{GaAs}\right)$. In the limit of zero temperature this Hall factor tends to 1 , which was implicitly shown by Langreth $^{32}$ for a bulk material. When we compare this result with the corresponding $3 \mathrm{D}$ case (dashed curve), $r_{H}$ attains a higher peak and has a more broadened shape. Notice that the position of the peak for the 2D case shifts to a higher temperature.

\section{B. The streaming state}

At sufficiently high electric fields, the conducting electrons in a semiconductor perform a streaming motion. In the streaming state an electron is accelerated by the electric field until its velocity reaches $v_{m} \geq v_{\text {LO }}$ and then it will emit a LO phonon. The electron velocity falls back

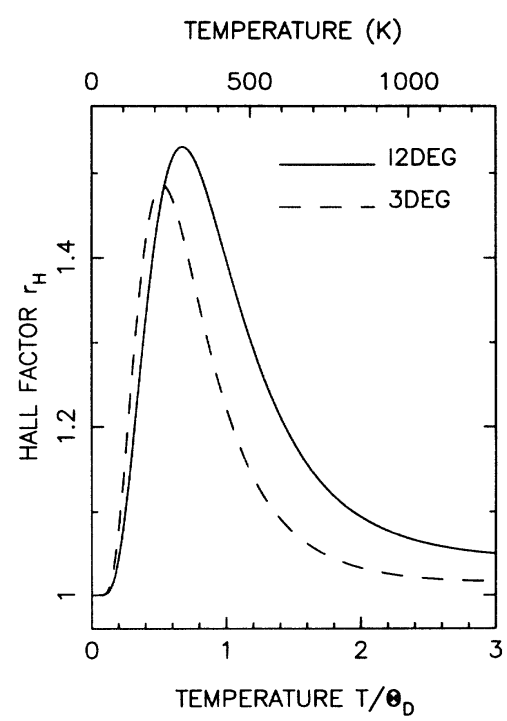

FIG. 5. The Hall factor in the limit of vanishing electric and magnetic fields as a function of temperature for the 2D (solid curve) and the $3 \mathrm{D}$ (dashed curve) case. $\Theta_{D}=\hbar \omega_{\mathrm{LO}} / k_{B}$ is the unit of temperature $\left(\Theta_{D}=425 \mathrm{~K}\right.$ for GaAs). The upper scale is the temperature scale for GaAs. 
around zero and the process of acceleration starts again. The penetration velocity $\mathbf{v}_{m}=\left(v_{m}^{x}, v_{m}^{y}\right)$ can be determined by the energy-balance equation ${ }^{28}$

$$
\langle\mathbf{v} \cdot \mathbf{F}\rangle=\int_{\hbar \omega_{\mathrm{LO}}}^{m^{*} v_{m}^{2} / 2} d E \lambda_{-}(E)=\hbar \omega_{\mathrm{LO}} \int_{1}^{\left(v_{m} / v_{\mathrm{LO}}\right)^{2}} d x \lambda_{-}(x),
$$

where $\lambda_{-}(x)$ is the scattering rate for LO-phonon emission and is given by Eq. (5) with $x=E / \hbar \omega_{\text {LO }}$. In the presence of a magnetic field the force is $\mathbf{F}=-e(\mathbf{E}+\mathbf{v} \times \mathbf{B})$. Setting $\Delta=\left(v_{m} / v_{\mathrm{LO}}\right)^{2}-1$, we obtain

$$
\frac{\left\langle v_{s}^{x}\right\rangle}{v_{\mathrm{LO}}}=-\alpha \xi \Delta\left(N_{0}+1\right) \frac{\omega_{\mathrm{LO}}}{\omega_{c}} \int_{0}^{\pi} \frac{d y}{1+\sqrt{1+\Delta \sin ^{2} y}}
$$

with $\mathbf{v}_{s}=\left(v_{s}^{x}, v_{s}^{y}\right)$ the average streaming velocity.

In the streaming state the average electron velocity after emitting a LO phonon is $\mathrm{v}_{0}=\left(v_{0}^{x}, v_{0}^{y}\right)$. Using Eq. (6b), which gives the probability to scatter an electron over an angle $\theta$ between its initial and final state, and noting $\left(v_{0} / v_{\mathrm{LO}}\right)^{2}=\left(v_{m} / v_{\mathrm{LO}}\right)^{2}-1=\Delta$, we obtain

$$
\begin{aligned}
\mathbf{v}_{0}=\langle|\mathbf{v}|\rangle \cdot \mathbf{e}_{v_{m}} & =v_{0} \mathbf{e}_{v_{m}} \int_{0}^{2 \pi} d \theta \cos \theta P(\theta) \\
& =\mathbf{v}_{m}\left[1-g^{-1}(\Delta)\right],
\end{aligned}
$$

where

$$
g(\Delta)=K\left[\left(\frac{\Delta}{1+\Delta}\right]^{1 / 2}\right] / E\left[\left(\frac{\Delta}{1+\Delta}\right]^{1 / 2}\right],
$$

and $E(x)$ is the complete elliptic integral of the second kind.

With Eqs. (13) and (3) we have, for the penetration velocities,

$$
\begin{aligned}
& v_{m}^{x} / v_{\mathrm{LO}}=-\left[1+\Delta-\xi^{2} g^{2}(\Delta) / 4\right]^{1 / 2}, \\
& v_{m}^{y} / v_{\mathrm{LO}}=-g(\Delta) \xi / 2 .
\end{aligned}
$$

The time $t_{s}$ an electron needs to reach a velocity $v_{m}$, starting from $v(t=0)=v_{0}$, is determined by the equation of motion (2),

$$
v_{x}^{2}\left(t_{s}\right)+v_{y}^{2}\left(t_{s}\right)=v_{m}^{2} .
$$

For the case of $\zeta>2$, Eq. (15) has no solution for $t_{s}$. This is due to the fact that LO-phonon emission scattering is not possible in the low electric- and high magnetic-field cases. After applying Eq. (13), Eq. (15) gives, for the fields dependence of $t_{s}$,

$$
\cos \left(\omega_{c} t_{s}\right)=\frac{\zeta^{2} g^{4}(\Delta) / 4-\left(1+\zeta^{2} / 4\right) g^{2}(\Delta)+\left(1 / \zeta^{2}-\frac{1}{2}-\Delta\right) g(\Delta)+1+\Delta}{1+\Delta-g(\Delta)+1 / \zeta^{2}} .
$$

For the case of zero penetration $\Delta=0$ one has $\cos \left(\omega_{c} t_{s}\right)=1-\zeta^{2} / 2$.

Thus we obtain for the average velocities for the streaming electrons

$$
\frac{\left\langle v_{s}^{x}\right\rangle}{v_{\mathrm{LO}}}=\frac{1}{v_{\mathrm{LO}} t_{s}} \int_{0}^{t_{s}} d t v_{x}(t)=-\frac{\zeta}{2} \frac{1}{\omega_{c} t_{s}},
$$

and

$$
\begin{aligned}
\frac{\left\langle v_{s}^{y}\right\rangle}{v_{\mathrm{LO}}} & =\frac{1}{v_{\mathrm{LO}} t_{s}} \int_{0}^{t_{s}} d t v_{y}(t) \\
& =-\frac{1}{\zeta}+\frac{\left[1+\Delta-\zeta^{2} g^{2}(\Delta) / 4\right]^{1 / 2}}{g(\Delta)} \frac{1}{\omega_{c} t_{s}} .
\end{aligned}
$$

In the absence of any scatterings, and for $\zeta \geq 2$, we have $\left\langle\mathbf{v}_{s}\right\rangle / v_{\mathrm{LO}}=(0,-1 / \zeta)$.

\section{The accumulation state}

In the accumulated state the electron motion is determined by the absorption of, and the consecutive emission of, LO phonons. It implies that the two-step scattering (absorption and emission) process is equivalent to quasielastic scattering. ${ }^{33}$ This can be described through the LO-phonon absorption scattering rate $\lambda_{+}(x)$. As a consequence the accumulation state is characterized by diffusive electron transport, i.e., a linearly increasing electron velocity $v_{a}$ with electric field. ${ }^{17}$ For electron motion in crossed electric and magnetic fields, the diffusion velocity $\mathbf{v}_{a}=\left(v_{a}^{x}, v_{a}^{y}\right)$ can be calculated with the aid of the Drude model ${ }^{34}$ Thus the average accumulation velocity $\left\langle\mathbf{v}_{a}\right\rangle=\left(\left\langle v_{a}^{x}\right\rangle,\left\langle v_{z}^{y}\right\rangle\right)$ is obtained by

$$
\frac{\left\langle v_{a}^{x}\right\rangle}{v_{\mathrm{LO}}}=-\frac{1}{\zeta} \frac{\omega_{c} \tau_{d}}{1+\left(\omega_{c} \tau_{d}\right)^{2}}
$$

and

$$
\frac{\left\langle v_{a}^{y}\right\rangle}{v_{\mathrm{LO}}}=-\frac{1}{\zeta} \frac{\left(\omega_{c} \tau_{d}\right)^{2}}{1+\left(\omega_{c} \tau_{d}\right)^{2}},
$$

where $\tau_{d}$ is the diffusion time determined by the LOphonon absorption scattering and is given by ${ }^{17}$

$$
\tau_{d}=\frac{1}{\alpha N_{0} \omega_{\mathrm{LO}}}\left(\pi \beta_{0}\right)^{-1 / 2} \frac{e^{-\beta_{0} / 2}}{K_{0}\left(\beta_{0} / 2\right)},
$$

with $\beta_{0}=\hbar \omega_{\mathrm{LO}} / k_{B} T$ and $K_{0}(x)$ the modified Bessel function of the second kind of order zero.

In general, a quasi-isotropic electron momentumdistribution function can be used to describe the electron accumulation motion, ${ }^{29}$ which is supported by our Monte Carlo simulations as presented in Sec. II. In Ref. 17 we showed that a displaced Maxwellian distribution could be chosen to describe the electron-velocity distribution in the diffusion state. In the accumulation state where electrons are within the passive regime we chose a displaced 
Maxwellian

$f(\mathbf{v})=\left[\frac{m^{*}}{2 \pi k_{B} T}\right]^{1 / 2} \exp \left[-\frac{m^{*}}{2 k_{B} T}\left(\mathbf{v}-\left\langle\mathbf{v}_{a}\right\rangle\right)^{2}\right]$,

centered around the accumulation velocity.

\section{The streaming-to-accumulation transition}

In the pure accumulation regime the electron orbit is so strongly curved that no LO phonon can be emitted, which occurs when $\left|v_{s}\right|<v_{\mathrm{LO}} / \zeta$. Noting that the electrons with velocity $|v|>\left|v_{s}\right|$ are not accumulated, we have for the relative number (or density) of accumulated electrons

$$
\begin{aligned}
n_{a} & =\int_{0}^{\left\langle v_{s}\right\rangle} d^{2} \mathbf{v} f(\mathbf{v}) / \int_{0}^{v_{\mathrm{LO}} / \zeta} d^{2} \mathbf{v} f(\mathbf{v}) \\
& =\int_{0}^{\left\langle v_{s}\right\rangle / v_{\mathrm{LO}}} d x F(x) / \int_{0}^{1 / \zeta} d x F(x),
\end{aligned}
$$

with $F(x)=x I_{0}\left(2 \beta_{0} x\left\langle v_{a}\right\rangle / v_{\text {LO }}\right) \exp \left(-\beta_{0} x^{2}\right)$, and $I_{0}(x)$ is the modified Bessel function. The rest of the electrons are in the streaming state and thus the relative density of the streaming electrons is

$$
n_{s}=1-n_{a} \text {. }
$$

Consequently, we obtain for the average drift velocity

$$
\begin{aligned}
& \left\langle v_{x}\right\rangle=n_{s}\left\langle v_{s}^{x}\right\rangle+n_{a}\left\langle v_{a}^{x}\right\rangle, \\
& \left\langle v_{y}\right\rangle=n_{s}\left\langle v_{s}^{y}\right\rangle+n_{a}\left\langle v_{a}^{y}\right\rangle .
\end{aligned}
$$

The relative density $n_{s}$ of the streaming electrons as a function of the electric field, or $1 / \xi$, is shown in Fig. 6(a) at a fixed magnetic field of $B=50 \mathrm{G}$ for different temper-

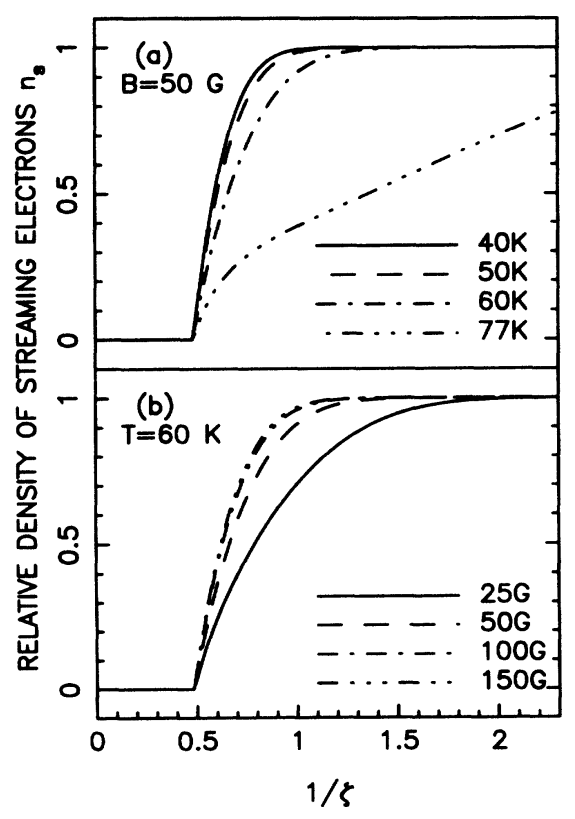

FIG. 6. The relative density of streaming electrons as a function of the electric field $1 / \xi \sim E / B$ as obtained from the present model: (a) for a fixed magnetic field of $B=50 \mathrm{G}$ and different temperatures, and (b) for a fixed temperature of $T=60 \mathrm{~K}$ and different magnetic fields.

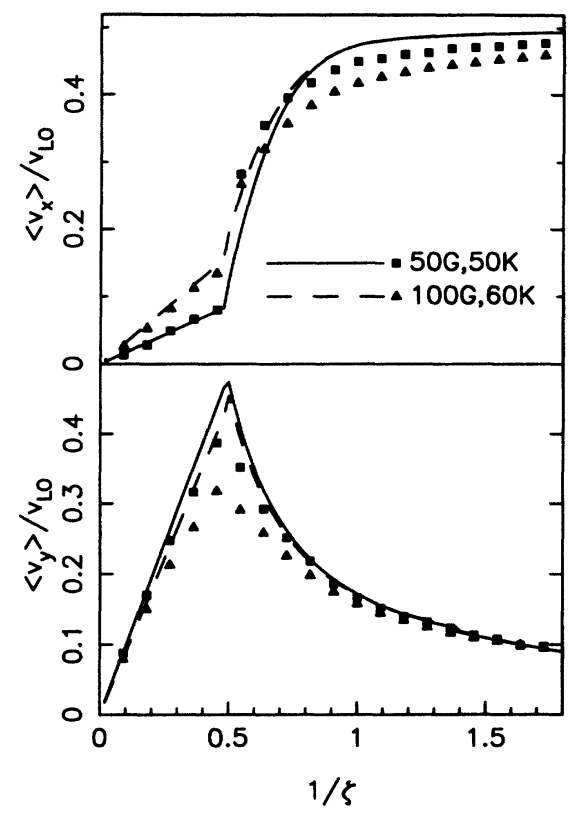

FIG. 7. The $x$ and $y$ components of the drift electron velocity as a function of the electric field as obtained from the present model (curves) are compared with the results obtained from a Monte Carlo simulation (symbols).

atures, and in 6(b) at a fixed temperature of $T=60 \mathrm{~K}$ for different magnetic fields. Three regimes can clearly be distinguished: (1) the accumulation regime $1 / \xi \leq 0.5$. The streaming motion is impossible, and thus $n_{s}=0 ;(2)$ the streaming-to-accumulation transition regime $0.5>1 / \xi<1$. $n_{s}$ increases rapidly with electric field; and (3) the streaming regime $1 / \zeta>1 . n_{s}$ depends very little

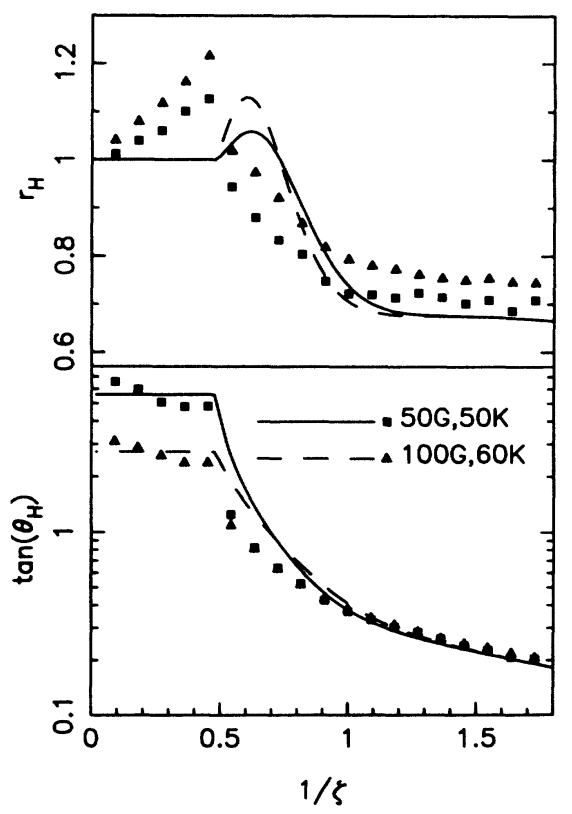

FIG. 8. The Hall factor $r_{H}$ and the tangent of the Hall angle $\tan \left(\theta_{H}\right)$ as a function of the electric field as obtained from the present model (curves) are compared to the results obtained from a Monte Carlo simulation (symbols). 
on the electric field and approaches 1 . The demarcations among these three regimes is smeared by increasing the temperature and/or lowering the magnetic field.

The average drift velocities $\left\langle v_{x}\right\rangle$ and $\left\langle v_{y}\right\rangle$, the Hall factor $r_{H}$, and the tangent of the Hall angle $\tan \left(\theta_{H}\right)$ versus electric field are shown in Figs. 7 and 8, respectively, as obtained with the present model and compared with our Monte Carlo simulation.

As is shown in Figs. 7 and 8, the three regimes are qualitatively described by the present model. The results for $\left\langle v_{x}\right\rangle,\left\langle v_{y}\right\rangle$, and $\tan \left(\theta_{H}\right)$ obtained with the model calculation show the same dependences on the electric field, the magnetic field, and temperature as obtained with the Monte Carlo simulation. The transition region is less well described by the present model. The reason is that the linear regime for $\left\langle v_{y}\right\rangle$ extends slightly to a higher electric field as compared to the Monte Carlo results, which leads to a poor agreement for the Hall factor $\left(r_{H}\right)$. The underlying reason is that in the present model LOphonon emission scattering becomes important, not at $\zeta \simeq 2$ but for $\zeta$ slightly less than 2 .

\section{SUMMARY}

We studied the transport of electrons in a very lowdensity two-dimensional system in the presence of crossed electric and magnetic fields. In a weak magnetic field, which is not strong enough to quantize the electron motion, the streaming-to-accumulation motion of electron was studied by (1) a Monte Carlo simulation and (2) a simple model calculation. The aim of this study was (1) to give a quantitative description of the electron motion in the presence of a weak magnetic field, (2) to develop further the streaming-to-accumulation model to the transport of electrons in a two-dimensional system in polar semiconductors, and (3) to assess the validity range of the present model for the $2 \mathrm{D}$ case by comparing with the Monte Carlo simulation and the 3D results.

We studied the dependence of the electron-momentum distribution function, the electron temperatures, the average drift electron velocities, the Hall factor, and the Hall angle on the electric field, the magnetic field, and the lattice temperature. When there is only scattering with LO phonons in an ideal 2D system, we found that (1) the streaming-to-accumulation transition model can be applied to describe the electron motion in two dimensions in crossed electric and magnetic fields, (2) the model is more suitable to the case of low lattice temperature and high magnetic field, and (3) in 2D the streaming-toaccumulation transition region is sharper than in the $3 D$ case.

\section{ACKNOWLEDGMENTS}

One of us (F.M.P.) is supported by the Belgian National Science Foundation, and W.X. was supported by the Supercomputer Project of NFWO (Nationaal Fonds voor Wetenschappelijk Onderzoek). This work was supported by Fonds voor Kollektief Fundamenteel Onderzoek (FKFO) Project No. 2.0093.91, by IUAP-11, and by "Diensten voor de Programmatie van het Wetenschapsbeleid" (Belgium) under Contract No. IT/SC/24.
*Also at University of Antwerp (RUCA), B-2020 Antwerpen and Technical University Eindhoven, NL-5600 Eindhoven, The Netherlands.

${ }^{1}$ See, e.g., Hot-Electron Transport in Semiconductors, edited by L. Reggiani, Topics in Applied Physics Vol. 58 (SpringerVerlag, Berlin, 1985).

${ }^{2}$ M. Sze, Physics of Semicondoctor Devices (Wiley, New York, 1981).

${ }^{3}$ See, e.g., Polarons and Excitons in Polar Semiconductors and Ionic Crystals, edited by J. T. Devreese and F. M. Peeters (Plenum, New York, 1984).

${ }^{4}$ T. Mimura, S. Hiyamizu, T. Fujii, and K. Nanbu, Jpn. J. Appl. Phys. 19, L225 (1980).

${ }^{5} \mathrm{~K}$. Hess and G. J. Iafrate, in Hot-Electron Transport in Semiconductors (Ref. 1), p. 201.

${ }^{6} \mathrm{~J}$. Shah, in The Physics of the Two-Dimensional Electron Gas, edited by J. T. Devreese and F. M. Peeters (Plenum, New York, 1987), p. 183.

${ }^{7}$ T. Ando, A. B. Fowler, and F. Stern, Rev. Mod. Phys. 54, 437 (1982).

${ }^{8}$ P. J. Price, Surf. Sci. 113, 199 (1982).

${ }^{9}$ B. K. Ridley, J. Phys. C 15, 5899 (1982).

${ }^{10}$ F. M. Peeters and J. T. Devreese, Phys. Rev. B 36, 4442 (1987).

${ }^{11}$ F. M. Peeters and J. T. Devreese, Solid State Phys. 30, 81 (1984).

${ }^{12}$ T. Kurosawa and H. Maeda, J. Phys. Soc. Jpn. 31, 668 (1971).

${ }^{13}$ See, e.g., G. D. Mahan, Phys. Rep. 145, 251 (1987).
${ }^{14}$ J. P. Leburton, J. Appl. Phys. 56, 2850 (1984).

${ }^{15}$ C. Jacoboni and L. Reggiani, Rev. Mod. Phys. 55, 645 (1983).

${ }^{16}$ F. M. Peeters, W. van Puymbroeck, and J. T. Devreese, Phys. Rev. B 31, 5322 (1985).

${ }^{17}$ W. Xu, F. M. Peeters, and J. T. Devreese, Phys. Rev. B 43, 14134 (1991).

${ }^{18}$ See, e.g., G. E. Alberga, R. G. van Welzenis, and W. C. de Zeeuw, Appl. Phys. A 27, 107 (1982).

${ }^{19}$ S. Komiyama, T. Masumi, and K. Kajita, Phys. Rev. B 20, 5192 (1979).

${ }^{20}$ See, e.g., G. Bauer, in Determination of Electron Temperatures and of Hot Electron Distribution Functions in Semiconductors, edited by G. Hohler, Springer Tracts in Modern Physics Vol. 74 (Springer-Verlag, Berlin, 1974), p. 1.

${ }^{21}$ J. T. Devreese and R. Evrard, Phys. Status Solidi B 78, 85 (1976).

${ }^{22}$ W. Xu, F. M. Peeters, and J. T. Devreese, J. Phys. Condens. Matter 3, 1783 (1991).

${ }^{23}$ F. Brosens and J. T. Devreese, Solid State Commun. 44, 597 (1982).

${ }^{24}$ W. Shockley, Bell Syst. Tech. J. 30, 990 (1950).

${ }^{25}$ S. Komiyama and R. Spies, Phys. Rev. B 23, 6839 (1981).

${ }^{26}$ P. Warmenbol, F. M. Peeters, and J. T. Devreese, Phys. Rev. B 33, 1213 (1986).

27 J. Sak, Phys. Rev. B 6, 3981 (1972).

${ }^{28}$ K. Seeger, Semiconductor Physics (Springer, New York, 1973).

${ }^{29}$ E. M. Conwell, High Field Transport in Semiconductors, Solid State Physics Suppl. 9 (Academic, New York, 1967). 
${ }^{30}$ Y. K. Pozhela, in Hot-Electron Transport in Semiconductors (Ref. 1), p. 113.

${ }^{31}$ F. A. Riddoch and B. K. Ridley, J. Phys. C 16, 6971 (1983).

${ }^{32}$ D. Langreth, Phys. Rev. 159, 717 (1967).
${ }^{33}$ E. J. Aas and K. Blotekjaer, J. Phys. Chem. Solids 35, 1053 (1974).

${ }^{34}$ See, e.g., G. Bastard, Wave Mechanics Applied to Semiconductor Heterostructures (Monographies de Physique, Paris, 1988). 